\title{
Profesorado de educación primaria y educación social ante la diversidad cultural: comparación de actitudes del alumnado entre España y Argentina
}

\author{
Rebolledo Gámez, Teresa \\ Universidad Pablo de Olavide, Sevilla, España \\ tjrebgam@upo.es
}

\begin{abstract}
Resumen
Las actitudes de los profesionales educativos ante las situaciones de diversidad se encuentran influenciadas por la formación inicial que reciben en el ámbito de la educación inclusiva. En este trabajo tenemos como objetivo analizar las actitudes del alumnado de las titulaciones de Profesorado en Educación Primaria y Educación Social ante la diversidad cultural, desde una perspectiva comparada entre España y Argentina. Partiendo de una metodología comparada, se ha implementado una encuesta al alumnado de último curso de ambas titulaciones en dichos países.

Los resultados obtenidos apuntan que la mayoría del alumnado no identificaba diversidad cultural con inmigración, ya que en ninguna de las titulaciones o países se hallaron porcentajes superiores al $50 \%$ de alumnado que indicara estar de acuerdo con esta afirmación. Sin embargo, exceptuando al alumnado de Educación Social en Argentina, en el resto de titulaciones y países, la mayoría del alumnado consideraba que la diversidad cultural hacía referencia a minorías étnicas.

Por ello, podemos concluir que la mayoría del alumnado de profesorado en Educación Primaria de España y Argentina, así como el alumnado de Educación Social de España, identificaba la diversidad cultural con colectivos étnicos minoritarios (sin incluir a las personas inmigrantes).

Este discurso sobre la diversidad cultural desde una perspectiva restringida conlleva el peligro de centrar las intervenciones para la atención a la diversidad cultural en «los otros», "los diferentes», fomentando acciones educativas, donde se enfatizan las diferencias (Barandica, 2006). De esta forma, se puede transmitir la idea de la diversidad cultural está en los demás mientras que los individuos locales son menos «étnicos» (Barquín, 2015). En contrapunto, las acciones educativas interculturales, por principio, se dirigen a todas las personas, colectivos e instituciones, independientemente de la presencia mayor o menor de minorías culturales y/o étnicas (Aguado, 2004; Barandica, 2006; Bartolomé, 2002).
\end{abstract}

\section{Abstract}

Attitudes of the educational professionals to diversity situations are influenced by the initial training in the scope of inclusive education. In this paper, we have aimed to analyze the attitudes in cultural diversity of students of primary education teachers and social education degrees, from a comparative perspective between Spain and Argentina. Based on a comparative methodology, it implemented a survey of students in the last year of both degrees in those countries.

The results indicate that the majority of students didn't identify diversity with immigration, since percentages greater than $50 \%$ of students indicating agreement with this statement were found in any of the degrees or countries. However, except for students of social education in Argentina, in the rest of degrees and countries, the majority of students considered that cultural diversity referred to ethnic minorities. Therefore, we can conclude that most students of primary education teachers in Spain and Argentina, as well as students of social education of Spain, identified cultural diversity with collective ethnic minority (not including immigrants people).

This discourse on cultural diversity from a restricted perspective brings the danger of focusing interventions for attention to cultural diversity on «the other», «different», promoting educational actions, which will emphasize the differences (Barandica, 2006). In this way, it can be transmitted the idea of cultural diversity is in others while local individuals are less " ethnic " (Barquin, 2015). In counterpoint, intercultural educational actions, by principle, are directed at all persons, groups and institutions, regardless of the more or less cultural and/or ethnic minority presence (Aguado, 2004; Barandica, 2006; Bartholomew, 2002).

Palabras clave: Actitudes, diversidad cultural, educación comparada, profesorado, educación social.

Keywords: Attitudes, cultural diversity, comparative education, teacher, social education. 


\section{INTRODUCCIÓN}

Las actitudes de los/as profesionales educativos ante las situaciones de diversidad se encuentran influenciadas por la formación inicial que reciben en el ámbito de la educación inclusiva. Para aproximarnos a este conocimiento, presentamos en este trabajo los resultados de una investigación que tiene como objetivo analizar las actitudes del alumnado de las titulaciones de Profesorado en Educación Primaria y Educación Social ante la diversidad cultural, desde una perspectiva comparada entre España y Argentina.

Conocer las percepciones del alumnado de las titulaciones de profesorado en Educación Primaria y Educación Social nos ayuda a determinar su posicionamiento frente al ámbito de la diversidad. Batsiou et al. (2008, citado en Boer et al., 2011), detectaban en su estudio que las actitudes docentes estaban influenciadas por la información y el conocimiento que el profesorado tenía sobre la inclusión, de modo que observó la existencia de una relación significativa entre la formación que el profesorado tenía sobre la atención a la diversidad y las actitudes que mostraban hacia la inclusividad.

La formación en diversidad cultural influye sobre las creencias que tiene el alumnado sobre esta realidad y que, a su vez, repercute en su futura labor profesional. Las actitudes de inhibición o pasividad ante esta realidad de diversidad cultural es una clave negativa si las escuelas quieren convertirse en espacios donde el aprender a convivir en la diferencia cultural sea un punto fundamental de las buenas prácticas docentes (Leiva, 2010).

Asimismo, en lo referente a la educación social, esta cuestión toma especial relevancia, en tanto posiblemente, uno de los ejes definitorios de la intervención de los/as educadores/as sociales en la actualidad sea la diversidad. Los ámbitos de intervención de los/as educadores/as sociales están impregnados de diversidad, como si ésta fuera un sello inherente a la intervención socioeducativa, porque pocas veces un/a educador/a trabaja con un grupo homogéneo y poco cambiante (Senent, 2011).

Una educación inclusiva implica una visión diferente de la educación basada en la diversidad y no en la homogeneidad (Blanco, 2008). Como punto de partida, en este apartado nos centramos en las percepciones que presentaba el alumnado de la titulación de Educación Social respecto al campo de la diversidad. Así, analizamos, entre otras cuestiones, la relación entre diversidad cultural y culturas minoritarias, además de las actitudes hacia la inclusión educativa, social y laboral de los colectivos culturalmente diversos.

\section{METODOLOGÍA}

Basándonos en una metodología de Educación Comparada, utilizamos como unidad de comparación el nivel estatal (Argentina y España). A partir de las fases planteadas por autores como Bereday (1964), Hilker (1962), García Garrido (1991), Ferrer (2002) o Llorent (2002), distinguimos cuatro fases en nuestra investigación: Identificación del problema ( $1^{\text {a }}$ fase); Estudio descriptivo y explicativo ( $2^{\mathrm{a}}$ fase); Yuxtaposición e interpretación de resultados ( $3^{\mathrm{a}}$ fase); y Estudio comparativo (4a fase). En este trabajo destacamos los resultados obtenidos fundamentalmente en las fases de yuxtaposición y estudio comparativo.

Tomando como referencia una metodología de corte cuantitativo, este estudio tiene como objetivo comparar las actitudes ante la diversidad cultural del alumnado de último curso de las titulaciones en Educación Social y Profesorado de Educación Primaria en España y Argentina.

Para ello, se implementó una encuesta, con un error muestral del 4,88\%, a 404 estudiantes matriculados/as en el último curso de dichas titulaciones en centros de carácter público, elegidos al azar en función de criterios de voluntariedad y disposición de las instituciones para su colaboración en este estudio. Concretamente, realizando un muestreo probabilístico estratificado, participaron 182 sujetos de Argentina y 222 de España. Por titulaciones, 290 participantes pertenecían a la titulación de Profesorado de Educación Primaria y 114, a Educación Social.

El instrumento utilizado fue un cuestionario, diseñado para este estudio y sometido a una doble validación: validación por personas expertas, tanto en metodología como en contenido; y posteriormente, la aplicación de un pretest a un grupo experimental que poseía características similares. 
En cuanto a la estructura del instrumento, además de un bloque previo con datos de identificación e información sociodemográfica, este cuestionario se componía por cinco dimensiones: 1) Percepciones del alumnado sobre el ámbito de la diversidad; 2) Competencias del alumnado para atender contextos de diversidad; 3) Formación del alumnado en el ámbito de la diversidad; 4) Necesidades formativas del alumnado en el ámbito de la diversidad; y 5) Propuestas formativas del alumnado en el ámbito de la diversidad.

Para este trabajo, nos centraremos en la primera dimensión, que, entre otras cuestiones, contenía cuestiones relativas a las actitudes del alumnado acerca de la diversidad cultural.

Cabe señalar que dicho cuestionario obtuvo un Alfa de Cronbach de 0,916 , lo que indicaba una alta fiabilidad, así como fue implementado de forma personal y online entre los años 2014 y 2015.

Los datos presentados en este trabajo son resultado de un análisis bivariante en el que se utilizó como prueba no paramétrica la $U$ de Mann-Whitney para dos muestras independientes. Por último, para el tratamiento y análisis de los datos se utilizó el programa SPSS (Statistical Package for the Social Sciences) versión20.

\section{ACTITUDES ANTE LA DIVERSIDAD CULTURAL DEL ALUMNADO DE PROFESORADO DE EDUCACIÓN PRIMARIA Y EDUCACIÓN SOCIAL EN ESPAÑA Y ARGENTINA}

En la presentación de los resultados obtenidos en este apartado de la investigación, realizamos una comparación entre países según la titulación observada.

El alumnado de las titulaciones de profesorado de Educación Primaria de España y Argentina mostró similitudes en cuanto a su desacuerdo sobre la vinculación de este ámbito con la inmigración y minorías étnicas, así como, en ambos casos, expresó su acuerdo general con la necesidad de intervenciones específicas en este campo.

Sin embargo, se hallaron diferencias significativas el ítem correspondiente a "La diversidad cultural enriquece la sociedad» $(U=11.244,000 ; p=0,012)$. Pese a que el grado de acuerdo con esta afirmación fue bastante alto en el alumnado de ambos países, era significativamente mayor en España $(M=4,6)$ que en Argentina $(M=4,27)$.

Aproximándonos a su posicionamiento en situaciones relacionadas con los colectivos minoritarios en distintos ámbitos (laboral, social o cultural), se encontraron diferencias significativas entre los resultados de Argentina y España en cuanto a los ítems que se detallan a continuación. En éstos se preguntaba su grado de acuerdo en cuanto a que las personas de culturas minoritarias debían:

a. Cumplir las normas de nuestro país $(U=11.484,000 ; p=0,040)$. El alumnado de las titulaciones españolas se encontraba más de acuerdo con esta afirmación $(M=4,48)$ que el de Argentina $(M=4,15)$, aunque cabe destacar que en ambos países se obtuvo un valor de media que indicaba bastante acuerdo hacia esta cuestión.

b. Tener igualdad de derechos que el resto de personas ( $U=11.436,000 ; p=0,024)$. En ambos países, el alumnado señalaba estar bastante de acuerdo con el reconocimiento de derechos de las personas de culturas minoritarias, estando significativamente más de acuerdo el alumnado de España $(M=4,69)$ que el de Argentina $(M=4,38)$.

c. Aprender la lengua oficial del país donde se encuentran $(U=13.017,000 ; p<0,001)$. Los/as estudiantes encuestados de España se mostraban más de acuerdo $(M=4,05)$ que el alumnado de Argentina $(M=3,52)$, con medio punto de diferencia entre las medias obtenidas. En cualquier caso, en los dos países de estudio, se encontraban de acuerdo con el deber de aprender la lengua oficial del lugar donde vivan los colectivos minoritarios.

d. Adaptarse a las costumbres sociales y culturales de la sociedad donde viven $(U=13.398,000 ; p<0,001)$. Mientras que, en Argentina, el alumnado de la titulación de profesorado en Educación Primaria expresaba su desacuerdo con la adaptación cultural y social $(M=2,84)$, en el caso de España, se mostraba de acuerdo $(M=3,56)$. 
e. Tener las mismas oportunidades laborales que el resto de personas del país donde viven $(U=11.799,000 ; p=0,003)$. Todo el alumnado de la titulación docente se encontraba bastante de acuerdo con esta cuestión, si bien se halló casi medio punto de diferencia entre las medias, siendo mayor en el caso de España $(M=4,49)$ que en Argentina $(M=4,06)$.

Por otra parte, respecto al alumnado de los estudios de Educación Social en España y Argentina, se encontraron diferencias significativas en determinadas afirmaciones relacionadas con la diversidad cultural. Por una parte, se hallaron diferencias en los siguientes ítems, relativos a que la diversidad cultural:

a. Se refiere a las personas inmigrantes $(U=1.110,000 ; p=0,044)$. El alumnado de Argentina mostraba un mayor grado de desacuerdo $(M=1,61)$ que el alumnado de España $(\mathrm{M}=2,19)$. Asimismo, la gran mayoría del alumnado de Argentina indicaba su desacuerdo, mientras que los resultados de España estaban más dispersos entre las opciones de respuesta. Este hecho podía deberse a que, a diferencia de Argentina, en España persiste la influencia de un discurso sobre interculturalidad europeo en el que existe una fuerte vinculación de la diversidad cultural con la inmigración (Carrasco,2015).

b. Enriquece la sociedad $(U=1.146,000 ; p=0,005)$. Todo el alumnado manifestaba un alto grado de acuerdo con esta cuestión, aunque era significativamente mayor en España $(M=4,72)$ que en Argentina $(M=4,33)$.

Entre los resultados en los que se encontraron similitudes entre países, se mostraba que el alumnado de la titulación de Educación Social no solía vincular la diversidad cultural con las culturas minoritarias, así como reconocía las aportaciones enriquecedoras de la diversidad cultural. También expresaba su acuerdo con la necesidad de intervenciones específicas en este ámbito.

Por otro lado, con respecto a la segunda agrupación de ítems sobre el ámbito de la diversidad cultural, se encontraron diferencias en relación a que las personas de culturas minoritarias deben:

a. Trabajar en los puestos vacantes que dejan las personas de las culturas mayoritarias o, en su caso, sociedad que los acoge $(U=597,000 ; p=0,013)$. En España, el alumnado indicaba estar en mayor desacuerdo $(M=1,56)$ que el alumnado de Argentina $(M=2,11)$.

b. Ser respetadas en cuanto a sus creencias religiosas o costumbres culturales $(U=1.138,500$; $\mathrm{p}=0,004)$. En ambos países, el alumnado mostró un alto grado de acuerdo, aunque era significativamente mayor en España $(M=4,78)$ que en Argentina $(M=4,39)$.

c. Cumplir las normas de nuestro país $(U=474,000 ; p=0,001)$. Tanto el alumnado de España como el de Argentina estaba de acuerdo con esta afirmación, si bien se obtuvo casi un punto de diferencia entre las medias de ambos países, siendo mayor el grado de acuerdo en el alumnado de Argentina ( $M=4,33)$ que en el de España $(M=3,53)$.

d. Tener las mismas oportunidades laborales que el resto de personas del país donde viven $(U=1.116,000 ; p=0,025)$. En esta afirmación se halló un punto de diferencia entre las medias de ambos países. El alumnado de España $(M=4,56)$ se mostraba bastante de acuerdo con este ítem, mientras el alumnado de Argentina estaba de acuerdo $(M=3,5)$. Destacaba que ningún/a estudiante de Educación Social de España manifestó su desacuerdo con esta cuestión.

e. Tener limitación de entrada al país a través de políticas públicas, si son extranjeros $(U=1.101,000 ; p=0,045)$. El alumnado de Argentina expresaba mayor desacuerdo $(M=1,5)$ que el de España $(M=1,88)$. Si bien en ambos países se reflejaba un alto grado de desacuerdo. 


\section{DISCUSIÓN Y CONCLUSIONES}

En general, el alumnado de los estudios docentes y de Educación Social, tanto en España como en Argentina, presentaba predisposición y actitudes positivas hacia la inclusión laboral y social de los colectivos minoritarios, así como hacia el reconocimiento de sus derechos sociales y culturales. Sin embargo, los resultados señalaban cierta tendencia hacia modelos asimilacionistas por parte del alumnado. Como puede verse, por ejemplo, en el grado de acuerdo mostrado hacia la adaptación a costumbres sociales y culturales de la sociedad mayoritaria.

Por otra parte, debemos destacar una conclusión fundamental. En nuestro estudio, se encontraron diferencias entre titulaciones y países en cuanto a la identificación de la diversidad cultural con colectivos minoritarios. Es numerosa la literatura científica que establece una estrecha vinculación de la diversidad cultural con los colectivos minoritarios, identificando un mayor grado de diversidad cultural con la presencia de inmigrantes o minorías étnicas en un contexto determinado. En España, es habitual encontrar estudios ligados a la inmigración como principal fuente de la diversidad cultural. Sin embargo, en América Latina, esta mirada desde la migración no es suficiente para debatir sobre interculturalidad, sino que se requiere prestar atención a la situación de los pueblos indígenas u originarios y la experiencia de dominación en la que han estado inmersos hasta la actualidad (Quintriqueo et al.,2014).

En España, el relativamente reciente fenómeno de la inmigración ha tenido un fuerte impacto en la sociedad y se ha generalizado un discurso de los/as profesionales donde la concepción de la diversidad cultural y la atención a la diversidad, en general, se relaciona frecuentemente con inmigrante o persona extranjera (Colectivo IOE, 2007). En nuestra investigación, se encontraba una diferenciación en concepciones en torno a la diversidad cultural e inmigración, según el país de estudio.

De esta forma, en la titulación de Educación Social, se hallaron diferencias significativas entre las medias obtenidas, identificándose diversidad cultural con personas inmigrantes, en mayor medida, en España que en Argentina.

Por otro lado, la mayoría del alumnado de profesorado en Educación Primaria indicó estar de acuerdo con que la diversidad cultural hacía referencia a minorías étnicas, tanto en España como en Argentina. En la titulación de Educación Social, se obtuvieron diferencias entre los porcentajes de alumnado que indicaba algún grado de acuerdo, siendo la mayoría del alumnado de España, pero no alcanzando la mayoría del alumnado de Argentina.

En definitiva, podemos decir que la mayoría del alumnado no identificaba diversidad cultural con inmigración, ya que en ninguna de las titulaciones o países se hallaron porcentajes superiores al $50 \%$ de alumnado que indicara estar de acuerdo con esta afirmación. Sin embargo, cuando se identificaba la diversidad cultural con minorías étnicas (tales como las personas de etnia gitana o de comunidades originarias, según el contexto), la situación era distinta. Exceptuando al alumnado de Educación Social en Argentina, en el resto de titulaciones y países, la mayoría del alumnado consideraba que la diversidad cultural hacía referencia a minorías étnicas.

Por todo ello, podemos concluir que la mayoría del alumnado de profesorado en Educación Primaria de España y Argentina, así como el alumnado de Educación Social de España, identificaba la diversidad cultural con colectivos étnicos minoritarios (sin incluir a las personas inmigrantes).

Este discurso sobre la diversidad cultural desde una perspectiva restringida conlleva el peligro de centrar las intervenciones para la atención a la diversidad cultural en "los otros», "los diferentes», fomentando acciones educativas, donde se enfatizan las diferencias (Barandica, 2006). De esta forma, se puede transmitir la idea de la diversidad cultural está en los demás mientras que los individuos locales son menos «étnicos» (Barquín, 2015).

En contrapunto, las acciones educativas interculturales, por principio, se dirigen a todas las personas, colectivos e instituciones, independientemente de la presencia mayor o menor de minorías culturales y/o étnicas (Aguado, 2004; Barandica, 2006; Bartolomé, 2002).

Cabe decir que queda mucho camino por recorrer y, aún hoy, encontramos un abismo entre la teoría sobre la interculturalidad y las escasas experiencias verdaderamente interculturales que se desarrollan en la práctica (Banks, 2009). Una práctica de la interculturalidad que debe tener presente el peligro de caer en una visión desprovista de conflicto en torno a la diversidad (Salinas y Lluch, 1996), no escondiendo las desigualdades sociales y educativas que subyacen en las relaciones entre culturas (Barquín, 2015). 


\section{REFERENCIAS BIBLIOGRÁFICAS}

Aguado Odina, T. (2004). Investigación en educación intercultural. Educatio Siglo XXI: Revista de la Facultad de Educación, 22, pp. 39-57.

Banks, J.A. (2008). An introduction to multicultural education. Boston, EEUU: Allyn and Bacon.

Barandica, E. (2006). Educación y multiculturalidad: análisis, modelos y ejemplos de experiencias escolares. En M.A. Essomba (Coord.), Construir la escuela intercultural. Reflexiones y propuestas para trabajar la diversidad étnica y cultural (pp. 15-20). Barcelona, España:Graó.

Barquín, A. (2015). ¿Qué debe hacer la escuela con las culturas familiares del alumnado inmigrante? Educar, 51 (2), $443-464$. Bartolomé Pina, M. (Coord.) (2002). Identidad y ciudadanía: un reto a la educación intercultural. Madrid, España: Narcea.

Bereday, G.Z.F. (1964). Comparative Method in Education. New York, EEUU: Holt, Rinehart \&Winston.

Blanco, R. (2008). Construyendo las bases de la inclusión y la calidad de la educación en la primera infancia. Revista de Educación, 347, 33-54.

Boer, A., Pijl, S.J. y Minnaert, A. (2011). Regular primary school teachers' attitudes towards inclusive education: a review of the literature. International Journal of Inclusive Education, 15 (3), 331-353.

Carrasco Carpio, C. (2015). Discurso de futuros docentes acerca de la diversidad intercultural. Papers, 100/2, 155-172.

Colectivo IOE (2007). Inmigración, género y escuela: exploración de los discursos del profesorado y del alumnado. Madrid, España: CIDE, Ministerio de Educación y Ciencia.

Ferrer Juliá, F. (2002). La educación comparada actual. Madrid, España: Ariel.

García Garrido, J.L. (1991). Fundamentos de Educación Comparada. Madrid, España: Dykinson. Hilker, F. (1962). Vergleichende Pädagogik. Eine Einführung in Ihre Geschichte, Theorie und. Praxis. Múnich, Alemania: Max Hueber.

Leiva Olivencia, J.J. (2010). La educación intercultural entre el deseo y la realidad: reflexiones para la construcción de una cultura de la diversidad en la escuela inclusiva. Revista Docencia e Investigación, 20, 149-182.

Llorent Bedmar, V. (2002). Educación comparada. Sevilla, España: Universidad de Sevilla. Quintriqueo, S., Quilaqueo, D., Lepe-Carrión, P., Riquelme, E., Gutiérrez, M. y Peña-Cortes, F. (2014). Formación del profesorado en educación intercultural en América Latina. El caso de Chile. Revista Electrónica Interuniversitaria de Formación del Profesorado, 17 (2), 201-217.

Salinas Catalá, J. y Lluch Balaguer, X. (1996). Uso (y abuso) de la interculturalidad. Cuadernos de Pedagogía, 252, 80-84.

Senent Sánchez, J.M (2011). Educación social: la diversidad como eje de trabajo en clave internacional. RES. Revista de Educación Social, 13, 1-19. 